\title{
Evolución histórica de la Fundación Universitaria Juan de Castellanos de Tunja 1963 - 2011.
}

Gerardo González Ramírez ${ }^{1}$

\begin{abstract}
Resumen
El presente artículo constituye parte integral del trabajo de tesis de Maestría en Historia, y tiene como objetivo principal indagar sobre el desarrollo histórico de la Fundación Universitaria Juan de Castellanos de Tunja durante los años 1963 - 2011. A través del análisis de fuentes primarias, secundarias y orales se pudo estudiar su origen, sus etapas, sus cambios más significativos y su contribución al desarrollo educativo regional y nacional.
\end{abstract}

Palabras clave: catequesis, educación, iglesia, religión, universidad. 


\title{
Historical evolution of University foundation Juan de Castellanos of Tunja (Colombia) 1963-2011.
}

\begin{abstract}
This study is an integral part of Master thesis in History. The main purpose was to research about the historical development of the foundation of University Juan de Castellanos of Tunja during the years 1963 - 2011. The university origin, stages and most important changes and its contribution to regional and national educational development was analyzed using primary and secondary sources and witnesses.
\end{abstract}

Key words: Catechesis, church, education, religion, university.

Recibido: 22 de agosto de 2013 Aceptado: 13 de diciembre de 2013

\section{lntroducción}

Este artículo presenta una descripción del origen, etapas, evolución y contribución al desarrollo educativo regional y nacional de la hoy Fundación Universitaria Juan de Castellanos de Tunja entre 1963 y 2011, que surge del interés y el esfuerzo pastoral de la Iglesia por fortalecer la educación religiosa y la instrucción catequística en un período donde el ideario político liberal colombiano cuestionaba su acción educativa y propendía por la libertad religiosa.

La construcción de la evolución histórica de la Fundación Universitaria se logró gracias a la revisión documental realizada en el Archivo Central de la Fundación, que permitió la recolección, clasificación, análisis e interpretación de las fuentes primarias. Fueron de vital importancia las comunicaciones de la Institución con la Arquidiócesis y el Ministerio de Educación Nacional (MEN), así como los informes y los planes de desarrollo presentados por los diferentes rectores. 
El análisis de estas fuentes permitió distinguir claramente tres etapas históricas: La primera etapa, denominada Instituto Catequístico Juan de Castellanos de (1963 a 1987), en que se analiza la enseñanza de la catequesis, los primeros cursos impartidos a dirigentes y catequistas parroquiales y a maestros normalistas superiores o bachilleres.

La segunda etapa, que explica el paso de Instituto Catequístico a Instituto Universitario de (1987 a 2002), se caracteriza por el surgimiento de los dos primeros programas de educación superior, con metodología a distancia, como son: La Licenciatura en Ciencias Religiosas, Ética y la Tecnología en Producción Agraria.

En la tercera etapa, que estudia el paso de Instituto Universitario a Fundación Universitaria a partir del año 2002, se amplía la cobertura de los programas, aumenta la población estudiantil y se analiza la importancia de las funciones de la educación superior en cuanto a extensión e investigación con miras al fortalecimiento de la calidad educativa y la prestación del servicio a nivel social.

\section{lglesia, política y educación}

La Iglesia como institución siempre ha estado ligada al desarrollo de las sociedades, no es una institución aislada. Como lo señala Ana María Bidegaín, "la Iglesia es policlasista y reproduce en su interior las contradicciones de la sociedad y las diferentes posturas ideológicas" (Bidegaín, 1983: 13). Gracias a su papel como agente evangelizador durante la colonia, como factor de cohesión en la Independencia y como elemento para fortalecer y consolidar la autoridad y el orden en la República, la Iglesia influyó y continúa influyendo en la vida social, política y educativa de los colombianos.

En el plano educativo, a través de la historia, el clero fue el dirigente natural de la educación confesional desde la colonia, para ellos la educación correspondía en primera instancia a los padres y en segundo lugar a la Iglesia hecho que constantemente era recalcado por la jerarquía eclesiástica. La educación para ser buena tenía que ser religiosa y para que fuese religiosa "debía ser confiada a hombres religiosos, porque sólo ellos enseñaban el conocimiento de Dios y su santa ley, que era la fuente donde emanaba toda justicia y toda moral" (Álvarez de Huertas, 1998: 261).

Sin embargo, esta concepción estaba supeditada a las relaciones entre la Iglesia y el Estado, que en Colombia se daba dentro del Patronato de 1824 que no contaba con el reconocimiento de la Santa Sede. Dicho patronato buscaba ejercer control sobre el poder político, económico y social de la Iglesia dentro de la sociedad, hecho que generó grandes conflictos como la guerra de los 
Supremos o de los Conventos entre 1839 y 1841, bajo la presidencia de José Ignacio de Márquez (1837 - 1841).

Por esta época se da en Colombia el surgimiento de los partidos políticos ${ }^{2}$, las reformas de mitad de siglo bajo la presidencia de José Hilario López (1849 $1853)^{3}$, las medidas anticlericales de Tomás Cipriano de Mosquera, la posterior desamortización de bienes de manos muertas y la firma del Concordato de 1887, que evidencian las diferencias entre partidos y con la Iglesia. En este marco se presentan "los enfrentamientos entre Iglesia católica y partido liberal colombiano, que llegaron a constituirse en frontera divisoria de los dos partidos tradicionales" (González, 2001: 344).

Este hecho llevaría a una serie de conflictos entre la Iglesia y el partido Liberal durante la primera mitad del siglo XX y que según algunos autores finalizaría con la imposición del Frente Nacional, como lo manifiesta los autores Cifuentes y Figueroa quienes afirman que: "superados los cruentos enfrentamientos entre liberales y conservadores, después de los años cincuenta con la consolidación del Frente Nacional, la institución eclesiástica hace las paces con los liberales" (2004: 386).

Después de este acuerdo, la Iglesia se dedicó a una labor de modernización interna, mostró una mayor preocupación por los problemas del desarrollo y la acción social. Sin embargo, el Frente Nacional tuvo una función negativa ya que se hizo para suavizar los enfrentamientos bipartidistas y no para legitimar un cambio positivo debido a que "Las estructuras eclesiásticas estaban hechas para la defensa frente al liberalismo laicizante y secularizante, pero no para una presencia creativa en la sociedad" (González, 2001: 301). Además, la Iglesia tuvo un retroceso y poca participación en las políticas educativas del Estado con relación a los periodos anteriores.

En este contexto nacional y durante los primeros años del Frente Nacional surge la iniciativa del Arzobispo de Tunja Ángel María Ocampo Berrío y del padre Álvaro Isaac Castillo Dueñas de crear una institución que permitiera promover la fe católica y renovar la moral de los fieles acorde con los objetivos del Concilio Vaticano II. Una manera de hacerlo era a través del cumplimiento del Plan de Enseñanza Religiosa adoptada por el Estado, de la enseñanza del Catecismo y de la instrucción catequística como medio de la educación cristiana. Con el derecho que se le otorgaba a la Iglesia de "establecer y dirigir libremente escuelas de cualquier orden y grado” (Pablo VI, 1965: 5), surge el Instituto Catequístico Juan de Castellanos con el fin de formar catequistas 
parroquiales y maestros normalistas superiores o bachilleres que promovieran la enseñanza de la religión católica.

\section{El Instituto Catequístico Juan de Castellanos 1963-1987}

La catequesis tiene su origen en la filosofía judeo-cristiana, su tema central es el "Kerygma" o mensaje salvador de Cristo, su enseñanza se daba a través del estudio del catecismo. Los primeros catecismos religiosos aparecen en el siglo VIII en la época de Carlo Magno, en el siglo IX aparece el catecismo cristiano del monje Ottiried y en el Concilio de Tortosa en 1429 se ordena la elaboración del primer catecismo para niños (Ocampo, 1999: 31). El método de enseñanza para la Religión Católica a través de la catequesis fue recomendado por el Concilio de Trento en 1546 y consistía en instruir a los alumnos, de una manera sencilla y práctica, a través de preguntas y respuestas. Actualmente se continúa con el mismo método de enseñanza con base en el Catecismo del Padre jesuita Gaspar Astete obra publicada en el año 1599, "la obra que más se ha editado en el mundo, llegando a más de 600 ediciones” (Ocampo, 1999: 32).

Con el tiempo, toda la renovación catequística de Europa se propagó a Hispanoamérica a través de la creación del Consejo Episcopal Latinoamericano CELAM, creado en 1955 para coordinar en escala continental los esfuerzos pastorales de la Iglesia. A los cinco años en 1960 se crea el Instituto Catequístico Latinoamericano ICLA con el fin de formar dirigentes nacionales y diocesanos los cuales, a su vez, formarían otros catequistas, tal es el caso del padre Álvaro Isaac Castillo Dueñas fundador del Instituto Catequístico Juan de Castellanos.

En Boyacá la catequesis se fortaleció aún más al ser elevada la Diócesis de Tunja a Arquidiócesis el 20 de junio de 1964 y bajo el gobierno de los tres arzobispos que ha tenido Tunja quienes han sido protagonistas de la evolución de la Fundación Universitaria Juan de Castellanos, son ellos:

\section{- Mons. Ángel María Ocampo Berrío. Primer Arzobispo de Tunja 1964-1970 \\ -Mons. Augusto Trujillo Arango. Segundo Arzobispo de Tunja 1970-1998 \\ -Mons. Luís Augusto Castro Quiroga. Tercer Arzobispo de Tunja a partir de1998}

A nivel institucional el primer documento, donde se habla de la instrucción catequística, que reposa en el Archivo Histórico de la universidad está fechado en Tunja el 22 de junio de 1960 y es una comunicación del Sr Canónigo Adán Puerto al obispo de Tunja Ángel María Ocampo Berrío donde le solicita que lo deje dedicarse a “...la instrucción catequística de los niños y el pueblo, encarecida por los cánones 1329-1336, en consonancia con el canon 711, 
\#2 C.S.J., reglamentados por la sagrada Congregación del Concilio en su decreto del 12 de Enero de 1935", El obispo de Tunja le responde el 6 de julio agradeciéndole su espíritu apostólico e informándole que "La Comisión de prelados encargada por la V Conferencia Episcopal, está preparando un plan de instrucción catequística y apologética para toda la nación",5.

Para la época hay un interés de la Iglesia por la formación de los laicos de conformidad con las directrices de la Santa Sede, pero también preocupación ya que no había una unidad en la enseñanza del catecismo en las diferentes Diócesis, se buscaba imponer un texto explicativo del catecismo para el uso de los maestros y su enseñanza en la instrucción primaria y secundaria. A nivel universitario se quería, de manera obligatoria, dictar conferencias religiosas y periódicas e imponer la asistencia de los alumnos.

Con miras a cumplir estos objetivos, se crea una oficina, en la Curia de Tunja, para la organización catequística en la Diócesis dirigida, durante casi dos años, por el Canónigo Adán Puerto y posteriormente asumida por el Padre Álvaro Isaac Castillo Dueñas. Teniendo en cuenta las orientaciones de la Comisión Episcopal de Catequesis con respecto a la necesidad de una pastoral catequística en Colombia, se resolvió crear institutos regionales que prestaran servicios a los obispos y párrocos en la formación y capacitación de apóstoles para la misión de enseñar.

Surge así el interés de fundar, en la ciudad de Tunja, un Instituto de Especialización de Religión a nivel universitario para normalistas y bachilleres que fueran después profesores de Religión en secundaria. Se comienzan a dar los cursos por ciclos con el control riguroso por parte del Ministerio de Educación Nacional (MEN). La primera promoción de este curso de profesores de religión contó con 17 mujeres estudiantes que terminaron el primer ciclo, 14 el segundo ciclo y 9 que terminaron los tres ciclos procedentes éstas últimas de los municipios de Rondón, Macanal, Susacón, Almeida, Somondoco y el Espino $^{6}$.

Ya habiendo transcurrido cinco años de trabajo catequístico en todas las parroquias de la Diócesis con sacerdotes, profesores, catequistas rurales y padres de familia y haberse preparado en la actividad de catequesis en la Pontificia Universidad Católica de Chile, se vuelve realidad el deseo del padre Álvaro Castillo Dueñas de crear el Instituto.

Monseñor Ángel María Ocampo Berrío, bajo el Decreto 639 de noviembre 4 de 1967, erige canónicamente el Instituto Catequístico Juan de Castellanos, también se decretó que la Junta directiva estaría formada por los arzobispos y

4 Archivo Histórico Instituto Universitario Juan de Castellanos. Folio 1.

5 Archivo Histórico Instituto Universitario Juan de Castellanos. Folio 2.

6 Archivo Histórico Instituto Universitario Juan de Castellanos. Folio 67. 
obispos residenciales en la Provincia Eclesiástica de Tunja y que la dirección estaría a cargo de un sacerdote, el padre Álvaro Castillo Dueñas, especializado en catequesis quien presentaría los estatutos y programas para su aprobación. Fue el primer Instituto Catequistico del país.

El Instituto inicia con el ofrecimiento de dos programas: El programa para dirigentes y catequistas parroquiales y el programa para maestros normalistas superiores o bachilleres. Desde su creación en 1963 hasta 1984 se realizaron 35 cursos para catequistas auxiliares de las parroquias para atender primeras comuniones, confirmaciones y orientaciones a comunidades, 350 cursos de actualización a profesores de enseñanza oficial primaria, 120 cursos de catequesis a jóvenes campesinos en Boyacá y 32 cursos de vacaciones para capacitación de profesores de religión de Educación Básica Primaria y de Educación Básica Secundaria, capacitando un total de 2030 profesores de 29 departamentos del país, especialmente Boyacá, Cundinamarca, Santander, Caquetá, Antioquia, Norte de Santander y Casanare; y de otras nacionalidades como Ecuador, Panamá, España, Venezuela, Italia, Puerto Rico, Canadá y Haití .

Gran parte del éxito del Instituto Catequístico Juan de Castellanos se debió al trabajo que realizaron las religiosas Hijas de la Iglesia, cuya Comunidad se vinculó al Instituto Catequístico en el año de 1963 y se retiró en el año 2001 después de 38 años de servicio en el campo espiritual y en las actividades de Extensión, Bienestar y Pastoral Universitaria. Actualmente la Fundación Universitaria continúa ofreciendo a la comunidad formación en catequesis bajo los principios y criterios del Directorio Nacional para la Catequesis en Colombia $^{8}$, y espacios para la reflexión y encuentro personal a través de la Pastoral universitaria y de la Oficina de Bienestar universitario que brinda apoyo en las diferentes líneas de desarrollo humano y servicio a la comunidad estudiantil.

\section{El Instituto Universitario Juan de Castellanos 1987-2002}

El Padre Álvaro Castillo Dueñas consideró que faltaba en el país un título universitario para los profesores de Religión, que los colocara a nivel profesional en igual categoría que los profesores especializados de las asignaturas del pensum oficial, ésta preocupación y el interés de los estudiantes de Pitalito (Huila) quienes pidieron elevar la capacitación catequística a categoría de Licenciatura, llevaron al Padre Castillo a adelantar conversaciones con el ICFES para concretar el programa de Licenciatura en Enseñanza Religiosa; con base en sus estudios en Roma proyectó el pensum universitario, diseñó el semáforo y los estatutos. Dos catedráticos del ICFES, ofrecieron su colaboración el 
Doctor Ernesto Vaca Perilla y el Doctor Julio Guzmán, ademas se contó con la asesoría de Galo Adán Clavijo y Francisco Mojica de Planeación Académica del ICFES (Castillo, 1993: 3).

Durante este proceso, Monseñor Augusto Trujillo Arango, Arzobispo de Tunja, por Decreto 743 del 30 de abril de 1985, reestructura el Instituto Catequístico como una Institución de Educación Superior con el nombre de Instituto Universitario Juan de Castellanos de la Arquidiócesis de Tunja, con la misión de impartir formación universitaria, de conformidad con la legislación colombiana y Canónica. Posteriormente, se obtuvo el reconocimiento civil como Institución de Educación Superior, por parte del MEN, mediante Resolución 2085 del 24 de marzo de 1987.

El 23 de octubre del mismo año, el MEN le aprobó los estatutos y el estudio de factibilidad y finalmente el 17 de diciembre por Acuerdo 256 del ICFES se autorizó ofrecer el nivel introductorio y se le concedió Licencia de Funcionamiento al programa de Licenciatura en Religión con la metodología a distancia hasta el 31 de diciembre de 1992; sin embargo el Instituto tenía 2 años para iniciar actividades de lo contrario perdería la licencia de funcionamiento.

Durante el año de 1988 se trabajó en la elaboración de estudios financieros y presupuestales para lograr el funcionamiento del Instituto y para las actividades de preapertura, se solicitó apoyo económico a la Arquidiócesis pero la respuesta fue negativa. Para no permitir que la licencia fuera cancelada, el padre Castillo, a finales de 1988, decide establecer un fondo por un millón de pesos de su propio pecunio para financiar los gastos iniciales.

Se decidió timbrar la papelería y hacer publicidad básicamente por correspondencia con los obispos, directores de catequesis, sacerdotes conocidos y exalumnos de los cursos vacacionales. Hubo una respuesta efectiva de 56 matriculados en Tunja y 17 en Pitalito para el primer programa ofrecido Licenciatura en Religión, ellos fueron los alumnos fundadores. El Instituto inició actividades universitarias el 4 y 5 de marzo de 1989 y como colaboradores, el padre Castillo designó al padre José Vicente Huertas Vargas para la Gestión Académica, al Doctor Julio Guzmán Roa como Secretario General y a la señorita Blanca Medina encargada de Admisiones, Registro y Control Académico?.

Una vez estructurados los criterios de planeación curricular, los componentes del plan de estudios, el diseño de instrucción y la organización para el trabajo académico de los diferentes programas con la metodología a distancia, el Instituto Universitario Juan de Castellanos exigió a sus tutores el cumplimiento de funciones del Manual del tutor, de William Botero, editado por el ICFES ${ }^{10}$ 
y se organizó un plan de formación de tutores como forma de cualificación docente para mejorar su desempeño como orientadores del proceso de aprendizaje de los estudiantes, el plan constaba de tres clases de cursos: cursos de inducción, cursos para manejo de contenidos y cursos de coordinación ${ }^{11}$.

Para el desarrollo de la metodología a distancia, el Instituto Universitario creó una estructura de apoyo con base en los Centros Regionales de Educación a Distancia CREAD y en los Círculos de Participación Académica y Social CIPAS activados por las Diócesis de los diferentes departamentos donde se hiciera presencia.

\section{Licenciatura en Religión}

Fue el primer programa académico aprobado en 1987 y ofrecido por el Instituto Universitario junto con el cual se crea la Facultad de Educación. El objetivo del programa se centró en preparar un Licenciado en Educación Religiosa y Ética para los niveles básico y medio de enseñanza; impulsar el propio crecimiento personal y católico y crear en el estudiante el liderazgo comunitario.

El plan de estudios de esta licenciatura se componía de 8 semestres cuya fundamentación se centraba en cuatro líneas:

- La Doctrinal que se basaba en los Estudios Bíblicos, Teología, Filosofía y Ética.

- La Catequística basada en la Historia de la catequesis, catequesis familiar y social, su metodología, el análisis de la legislación Canónica, la Doctrina social de la Iglesia y la Liturgia.

- La Pedagógica que comprendía la Psicología general, evolutiva y del aprendizaje, la Historia de la pedagogía, Teoría de la educación, legislación y administración escolar y diseño curricular. Además la práctica docente que se realizaba en los dos últimos semestres académicos.

- La de Liderazgo que incluía sociología general, participación $y$ desarrollo familiar, escolar y comunitario, trabajo cientifico, metodologías y técnicas de investigación, técnicas de comunicación e intervención, estadística y la elaboración de una Tesis en el último semestre académico.

A partir de su apertura, el programa de Licenciatura en Ciencias Religiosas y Ética tuvo gran acogida, desde 1990 a 1993 el incremento en la población 
estudiantil fue de más del 227\%, siendo reconocido el Instituto Universitario Juan de Castellanos como uno de los más importantes en la formación de docentes de Religión en Boyacá.

La graduación de las primeras promociones se efectuó, el 10 de diciembre de 1993, en la Sede Central del Seminario Mayor en Tunja con 29 graduandos y el 19 de diciembre del mismo año en el Templo parroquial de Valvanera Nueva en Pitalito con 21 graduandos, para un total de 50, bajo la rectoría del padre José Vicente Huertas Vargas. A propósito de la graduación, el Padre Álvaro Isaac Castillo Dueñas en su discurso para los graduandos, desde su natal Somondoco, escribe: “'HOY ES FESTÍN DE LA CATEQUESIS! Es la vendimia de frutos de una larga espera; Hoy es la cosecha celebrada entre cantares de júbilo. Una cosecha de 50 Licenciados para la evangelización en Colombia. FELICITACIONES! Están de plácemes las diócesis de los graduados "12.

El programa de Licenciatura en Ciencias Religiosas y Ética ya ha graduado a 659 estudiantes y actualmente cuenta con 276 matriculados (2011); este programa ha sido un baluarte en la Institución y es uno de los principales promotores de la creación de espacios para el análisis y reflexión en torno a la enseñanza de la Religión y la Ética en los tiempos modernos.

\section{Tecnología en Producción Agraria}

Es el segundo programa que se creó en el Instituto Universitario Juan de Castellanos por Acuerdo 026 del 1 de agosto de 1994 emitido por el Consejo Superior, con metodología semipresencial en las poblaciones de Guateque, Toca, Paipa, Ramiriquí, Soatá, Tibaná, Santa Sofía, Villa de Leiva y Puerto Boyacá, bajo la dirección del ingeniero agrónomo Pedro José Almanza Merchán y la colaboración del ingeniero José Francisco García Molano.

El objetivo del programa era formar tecnólogos competentes y empresarios agrarios que, con compromiso personal de ser auténticos colombianos católicos, promovieran investigaciones y aplicaciones de tecnologías limpias y lideraran el cambio positivo de la técnica y de la comunidad.

El plan de estudios comprendía seis semestres académicos, durante los tres primeros el estudiante recibía cinco asignaturas por semestre, como soporte fundamental de carácter académico en las Ciencias Básicas y en la investigación. Con las Prácticas Agronómicas Integrales I, II y III el estudiante se iniciaba en las distintas actividades agropecuarias que bien podía realizarse en una granja de la región y/o en las fincas de los estudiantes.

En el cuarto y quinto semestre el estudiante recibía asignaturas de mayor especialización agronómica desde el punto de vista académico y Producción 
Agrícola y Animal I y II desde el punto de vista práctico, correspondientes a la elaboración de proyectos específicos de carácter empresarial.

En 1995, el programa de Tecnología en Producción Agraria, inició sus actividades con 57 estudiantes en las sedes de Ramiriquí y Toca, contando para 1998 con 139 estudiantes en todas sus sedes y en marzo del mismo año recibieron el título de Tecnólogos en Producción Agraria, 38 estudiantes ${ }^{13}$. La propuesta de la Tecnología era de una agricultura para la vida, por eso su producción agrícola y pecuaria era netamente orgánica. Su trabajo fue proyectado a nivel regional y nacional.

\section{La Fundación Universitaria Juan de Castellanos a partir de 2002}

Una vez consolidadas las Facultades de Ciencias de la Educación con el programa de Licenciatura en Ciencias Religiosas y Ética y la Facultad de Ciencias Agrarias con Medicina Veterinaria e Ingeniería Agropecuaria, se inicia un proceso de restructuración en la Fundación y se amplía la cobertura en otras áreas del conocimiento con la creación de nuevos programas académicos. El 5 de agosto de 2002 el MEN, mediante la resolución 1904, le dio aprobación a los nuevos estatutos y le otorgó el nombre de Fundación Universitaria Juan de Castellanos.

En la actualidad, segundo semestre de 2011, la Fundación Universitaria cuenta con siete Facultades, nueve programas de pregrado y cuatro programas de posgrado como se muestra en la tabla No. 1.

Tabla 1: Programas ofrecidos por la Fundación Universitaria.

\begin{tabular}{|c|c|c|}
\hline FACULTAD & PROGRAMAS DE PREGRADO & PROGRAMAS DE POSGRADO \\
\hline \multirow[b]{2}{*}{$\begin{array}{l}\text { CIENCIAS DE } \\
\text { LA EDUCACIÓN, } \\
\text { HUMANIDADES, } \\
\text { FILOSOFIA YARTES } \\
\text { Acuerdo } 088 \text { del } 24 \text { de } \\
\text { julio de } 1998\end{array}$} & $\begin{array}{l}\text { LICENCIATURA CIENCIAS } \\
\text { RELIGIOSAS }\end{array}$ & \multirow{2}{*}{$\begin{array}{c}\text { ESPECIALIZACION EN LUDICA } \\
\text { EDUCATIVA } \\
\text { ESPECIALIZACION EN ETICAY } \\
\text { PEDAGOGIA } \\
\text { ESPECIALIZACION EN PLANEACION } \\
\text { EDUCATIVA Y PLANES DE } \\
\text { DESARROLLO }\end{array}$} \\
\hline & $\begin{array}{c}\text { LICENCIATURA EDUCACIÓN } \\
\text { FÍSICA }\end{array}$ & \\
\hline \multirow{2}{*}{$\begin{array}{c}\text { CIENCIAS AGRARIAS } \\
\text { Acuerdo } 128 \text { del } 24 \text { de } \\
\text { noviembre de } 1999\end{array}$} & MEDICINA VETERINARIA & \multirow{4}{*}{$\begin{array}{l}\text { ESPECIALIZACION EN SEGURIDAD DE } \\
\text { LA INFORMACION * }\end{array}$} \\
\hline & INGENIERÍAAGROPECUARIA & \\
\hline \multirow{2}{*}{$\begin{array}{c}\text { INGENIERÍA } \\
\text { Acuerdo } 129 \text { del } 24 \text { de } \\
\text { enero de } 2000\end{array}$} & INGENIERÍA DE SISTEMAS & \\
\hline & $\begin{array}{l}\text { TECNOLOGÍA EN } \\
\text { ELECTRÓNICA }\end{array}$ & \\
\hline
\end{tabular}




\section{Gerardo González Ramírez}

Evolución histórica de la Fundación Universitaria Juan de Castellanos de Tunja 1963 - 2011. Artículo producto de la investigación.

\begin{tabular}{|c|c|c|}
\hline FACULTAD & PROGRAMAS DE PREGRADO & PROGRAMAS DE POSGRADO \\
\hline $\begin{array}{c}\text { CIENCIAS SOCIALES Y } \\
\text { ECONOMICAS }\end{array}$ & $\begin{array}{c}\text { TRABAJO SOCIAL } \\
\text { CONTADURIA PÚBLICA } \\
\text { noviembre de } 2001\end{array}$ & $\begin{array}{c}\text { CONTADURIA PÚBLICA } \\
\text { NOCTURNO }\end{array}$ \\
\hline $\begin{array}{c}\text { CIENCIAS JURIDICAS } \\
\text { Y POLITICAS }\end{array}$ & DERECHO DIURNO \\
\hline $\begin{array}{c}\text { INTERNACIONALES } \\
\text { Acuerdo } 264 \text { del } 29 \text { de } \\
\text { abril de } 2009\end{array}$ & DERECHO NOCTURNO \\
\hline
\end{tabular}

En las siguientes tablas se ven las preferencias de los estudiantes frente a los programas que ofrece la Fundación Universitaria Juan de Castellanos.

Tabla 2

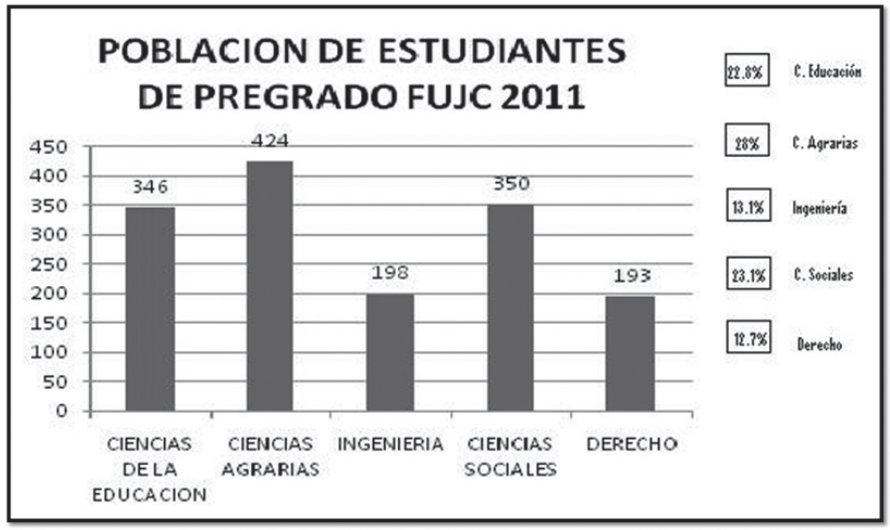

Hay una población activa de 4089 estudiantes: 1511 de pregrado que representan el $36.95 \%$ y 2578 de posgrado que representan el $63 \%$ del total de estudiantes de la Fundación. El programa con mayor demanda es el de Medicina Veterinaria de la Facultad de Ciencias Agrarias con 333 estudiantes.

Tabla 3

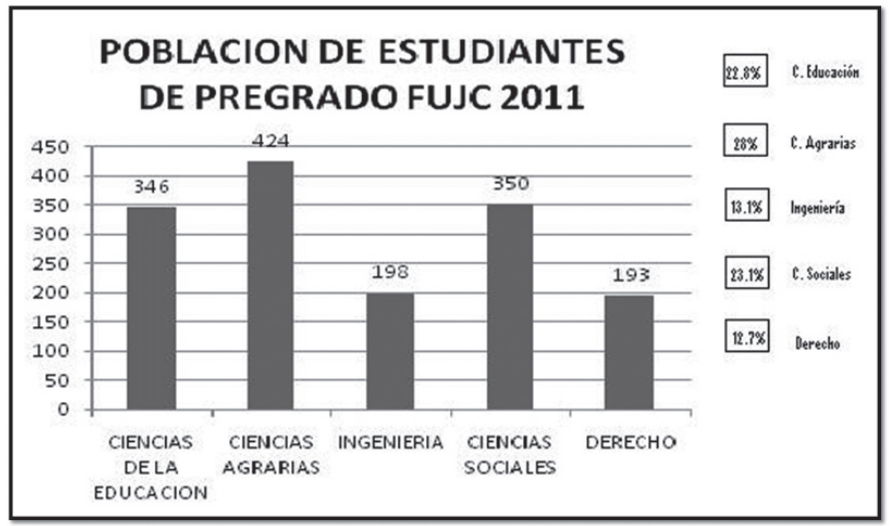

Elaborado por el autor a partir de la información recolectada de la Oficina de Registro y Control. Octubre 2011 
Frente a la política institucional de seguimiento a la población de egresados, no se tiene uno real, ni un observatorio laboral por lo tanto es difícil reconocer su impacto en el medio social y académico.

Dentro del desarrollo de este trabajo, y a pesar de las dificultades para ubicar egresados, se logró aplicar una encuesta a 182 exalumnos de diferentes promociones de los programas de pregrado y posgrado, cuyo objetivo era conocer el impacto que ha tenido la profesión en su vida personal, laboral y académica. Se elaboraron siete preguntas que respondían a cinco categorías: laboral, disciplinaria, investigativa, de calidad y de impacto social. Su análisis se presenta en la tabla No. 4.

Tabla 4 Matriz de la encuesta a egresados

\begin{tabular}{|c|c|c|}
\hline CATEGORIAS & PREGUNTAS & ANALISIS E INTERPRETACION \\
\hline LABORAL & $\begin{array}{l}\text { 1. ¿Desde su campo laboral } \\
\text { siente que la formación recibida, } \\
\text { en la Fundación, ha contribuido } \\
\text { al desarrollo humano dentro del } \\
\text { contexto que usted escogió? }\end{array}$ & $\begin{array}{l}\text { La formación recibida ha contribuido al } \\
\text { desarrollo humano integral, se enfatiza } \\
\text { en la formación ética humanística, } \\
\text { el fortalecimiento de valores y la } \\
\text { responsabilidad social, hechos que han } \\
\text { contribuido a desenvolverse mejor en el } \\
\text { ámbito social y que facilita la interacción } \\
\text { con las familias, pacientes y demás grupos } \\
\text { sociales. } \\
\text { La formación académica: teórica y práctica } \\
\text { recibida, ha sido la base principal para un } \\
\text { desempeño profesional y laboral óptimo. }\end{array}$ \\
\hline DISCIPLINAR & $\begin{array}{l}\text { 2. ¿El programa lo preparó } \\
\text { en las área de conocimiento } \\
\text { pertinentes de su formación } \\
\text { disciplinar, para ser competente } \\
\text { laboralmente? }\end{array}$ & $\begin{array}{l}\text { Los egresados manifiestan que la estructura } \\
\text { curricular permite la interdisciplinariedad y } \\
\text { se articula a las diferentes carreras, hecho } \\
\text { que ha fortalecido sus competencias básicas } \\
\text { y laborales: en la intervención social, en } \\
\text { el cuidado de pacientes, en la solución de } \\
\text { conflictos y en la atención y orientación de } \\
\text { víctimas de la violencia. Sin embargo, se } \\
\text { ve una tendencia en la falta de prácticas } \\
\text { en el programa de Medicina veterinaria y } \\
\text { en la poca profundización en el área de } \\
\text { programación de la carrera de Ingeniería de } \\
\text { Sistemas. }\end{array}$ \\
\hline INVESTIGATIVO & $\begin{array}{l}\text { 3. ¿La formación profesional } \\
\text { obtenida en la Universidad } \\
\text { aportó elementos investigativos } \\
\text { que generaron impacto social? }\end{array}$ & $\begin{array}{l}\text { Los egresados sienten que recibieron en } \\
\text { su formación profesional elementos sobre } \\
\text { investigación en las diferentes carreras que } \\
\text { ofrece la Fundación Universitaria Juan de } \\
\text { Castellanos. Los egresados de Ingeniería } \\
\text { de Sistemas no lo percibieron así, porque } \\
\text { al momento de graduarse (años: 2007, } \\
2008 \text { y 2009) no se habían consolidado } \\
\text { los grupos de investigación. Al respecto } \\
\text { es importante resaltar los esfuerzos que } \\
\text { ha hecho la Fundación Universitaria para } \\
\text { consolidar la red de inicien en las diferentes } \\
\text { Facultades, particularmente en Ciencias de } \\
\text { la Información con la creación de los grupos } \\
\text { de investigación: GPL@I+D y GEATIC. }\end{array}$ \\
\hline
\end{tabular}




\section{Gerardo González Ramírez}

Evolución histórica de la Fundación Universitaria Juan de Castellanos de Tunja 1963 - 2011. Artículo producto de la investigación.

\begin{tabular}{|c|c|c|}
\hline CALIDAD & $\begin{array}{l}\text { le } \\
\text { 4. ¿El programa del cual es } \\
\text { egresado tiene oferta laboral en } \\
\text { el campo profesional? } \\
\text { ¿Por qué? ¿Qué sugiere para } \\
\text { implementar y mejorarlo? } \\
\text { 5. ¿La calidad del programa } \\
\text { se ajusta a los estándares } \\
\text { profesionales internacionales de } \\
\text { calidad comparados con otros } \\
\text { de la misma naturaleza? ¿Por } \\
\text { qué? }\end{array}$ & $\begin{array}{l}\text { Frente a la calidad de los programas, los } \\
\text { egresados manifiestan que se sienten } \\
\text { competentes en el campo profesional } \\
\text { y laboral; que la educación recibida es } \\
\text { de calidad, situación evidente en los } \\
\text { campos donde se desempeñan: salud, } \\
\text { administración, sistemas, investigación e } \\
\text { intervención por proyectos. } \\
\text { Coinciden en que hay oferta laboral para } \\
\text { las diferentes profesiones, sin embargo } \\
\text { plantean como limitante la competencia y la } \\
\text { exigencia de experiencia laboral. } \\
\text { Proponen como sugerencias para el } \\
\text { mejoramiento profesional: la profundización } \\
\text { y renovación de los programas académicos, } \\
\text { reforzar el ejercicio interdisciplinario en } \\
\text { salud mental, ofrecer más programas de } \\
\text { posgrado, mejorar las prácticas, crear } \\
\text { convenios interinstitucionales, fortalecer el } \\
\text { manejo del Inglés como segunda lengua, } \\
\text { mejorar el seguimiento a egresados y formar } \\
\text { en emprenderismo para crear empresa. } \\
\text { Los entrevistados argumentaron que no } \\
\text { conocen los estándares internacionales de } \\
\text { sus respectivas profesiones. Sin embargo, } \\
\text { manifestaron que están satisfechos con la } \\
\text { educación impartida en la Fundación. }\end{array}$ \\
\hline IMPACTO SOCIAL & $\begin{array}{l}\text { 6. ¿Los aportes académicos } \\
\text { y el valor social agregado de } \\
\text { la carrera ha marcado una } \\
\text { diferencia con otros programas } \\
\text { de la misma denominación que } \\
\text { existe en la región y en el país? } \\
\text { ¿Por qué? } \\
\text { 7. ¿Desde su profesión como } \\
\text { ve en la actualidad el progreso } \\
\text { que ha tenido el programa y la } \\
\text { Universidad? ¿Cuál sería su } \\
\text { aporte? }\end{array}$ & $\begin{array}{l}\text { El valor agregado de la Fundación } \\
\text { Universitaria Juan de Castellanos es la } \\
\text { formación del SER, la deontología de las } \\
\text { profesiones, la formación en valores como } \\
\text { lo expresa su lema: "por la Civilización del } \\
\text { amor". } \\
\text { Como fortaleza también está la } \\
\text { infraestructura de la Fundación: laboratorios, } \\
\text { salas tecnológicas y de electrónica, } \\
\text { biblioteca, la clínica Francisco de Asís } \\
\text { en Soracá, la calidad de los docentes, el } \\
\text { reconocimiento de Ciencias Agrarias y } \\
\text { Medicina Veterinaria como las mejores del } \\
\text { departamento y del país, la existencia del } \\
\text { programa de Trabajo Social con énfasis en } \\
\text { ruralidad único en el suroriente colombiano y } \\
\text { La aparición de nuevas carreras de pregrado } \\
\text { y posgrado. } \\
\text { La Fundación Universitaria Juan de } \\
\text { Castellanos se proyecta como una de las } \\
\text { mejores de la región, busca ser Universidad } \\
\text { y tiene proyectado ser Pontificia. } \\
\text { El aporte que harían sería vincularse a } \\
\text { programas de posgrado y ayudar a su } \\
\text { promoción, aportar su conocimiento y } \\
\text { experiencia profesional a los procesos } \\
\text { internos de la universidad. }\end{array}$ \\
\hline
\end{tabular}


Las políticas con respecto a los egresados y su impacto en el medio garantizan los procesos académicos y la eficiencia del profesional a través de su producción intelectual y del reconocimiento social, sin embargo, es de vital importancia consolidar la oficina de egresados, ampliar el registro de egresados, su ubicación profesional, índice de empleo y darles mayor participación en los procesos de evaluación y prospección del programa del cual son egresados.

\section{Extensión Universitaria}

La Unidad de Extensión Universitaria es la encargada de "promover y ejecutar los servicios de capacitación y asesoría académica" "14 a través de ella la Fundación Universitaria Juan de Castellanos busca constantemente promover e impulsar el desarrollo social, cultural y económico de la región, no sólo mediante la educación que imparte, sino también mediante la ejecución de proyectos de alto impacto.

La Extensión Universitaria comprende los programas de Educación continuada, servicios de consultoría técnico científicos, gestión tecnológica, asesorías educativas, prácticas académicas, seminarios, cursos de idiomas, diplomados ${ }^{15}$ y otros, destinados a la difusión de conocimientos, al intercambio de experiencias, así como a las actividades de servicios tendientes a procurar el bienestar general de la comunidad y la satisfacción de las necesidades de la sociedad.

\section{lnvestigación}

La investigación de la Fundación Universitaria Juan de Castellanos se concibe "como un proceso de generación de conocimiento articulado a la función docente, que se fundamenta en las potencialidades y limitaciones del desarrollo local, regional y nacional en un contexto globalizado. Contribuye a la construcción de una cultura cientifica e investigativa institucional que garantice la calidad educativa, mediante el desarrollo de la capacidad de análisis, comprensión, apropiación, aplicación e innovación y creación de conocimiento"'t6.

En el campo investigativo el Instituto Universitario Juan de Castellanos creó el Instituto de Investigaciones Científicas INICIEN ${ }^{17}$. La identidad científica de

\footnotetext{
14 Fundación Universitaria Juan de Castellanos. Consejo Superior. Acuerdo 169 del 1 de noviembre de 2001. Por el cual se determina la estructura orgánica de la Fundación Universitaria Juan de Castellanos. p. 15

15 Fundación Universitaria Juan de Castellanos. Consejo Superior. Acuerdo 312 del 6 de diciembre de 2011. Por el cual se expide el Plan de Desarrollo periodo 2012-2019 p. 47-48

16 Fundación Universitaria Juan de Castellanos. Consejo Superior. Proyecto Educativo Universitario. Acuerdo 220 del 8 de agosto de 2005. Artículo 16. p. 10.
}

17 Fundación Universitaria Juan de Castellanos. Consejo Superior. Acuerdo 095 del 25 de febrero de 1999. 
INICIEN se centra en la formación de las nuevas generaciones y la orientación de actividades complementarias de la investigación científica y el desarrollo tecnológico.

La nueva propuesta es INICIEN - RED que está conformada por seis Institutos de Investigaciones Científicas y 18 grupos de investigación como los núcleos o unidades básicas del Sistema Nacional de Ciencia Tecnología e Innovación SNCTI para la generación de conocimiento a partir de la investigación, sus miembros formulan uno o varios problemas de su interés, trazan un plan estratégico de largo o mediano plazo para trabajar en él y producen unos resultados de conocimiento sobre el tema cuestión. La tabla 5 presenta la estructura de INICIEN de la Fundación Universitaria.

Tabla 5 ESTRUCTURA DE INICIEN.

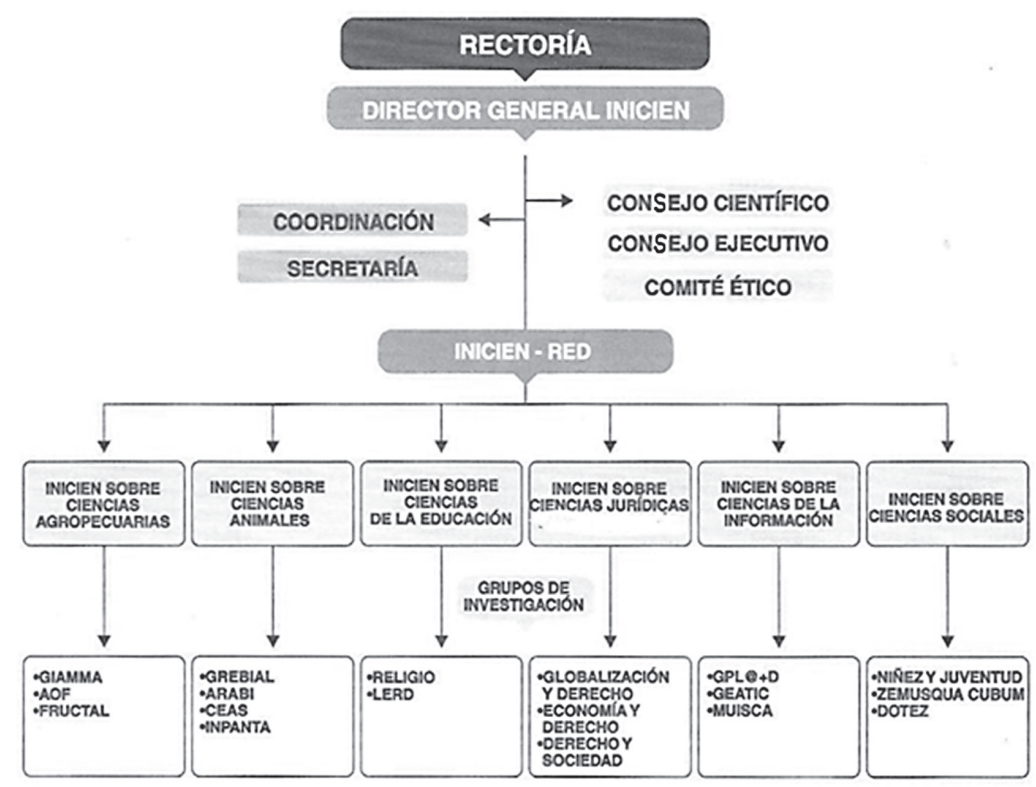

Fuente: Organigrama de INICIEN. Proyección Científica 2012. p. 51.

La investigación como función social de la Fundación es un eje transversal que motiva a directivos, docentes y estudiantes a la búsqueda y producción continua del conocimiento por medio de planes y proyectos que cada uno de los programas demuestra a través de las cuatro revistas científicas que tiene: Cultura Científica, Conexión Agropecuaria JDC, Educación y Territorio y Desarrollo, Economía y Sociedad.

La mayor producción de artículos de investigación es de los institutos de investigaciones científicas de Ciencias Agrícolas, Ciencias Veterinarias y 
Ciencias de la Información. Cabe resaltar que el grupo de investigación Denominación de origen TERROIR ${ }^{18}$ y zonificación DOTEZ, de Ciencias Agrícolas, tiene una amplia investigación a partir del Proyecto de Viticultura tropical de calidad que se inició en la Loma de Punta Larga ${ }^{19}$, en el Valle del Sol (Sogamoso) en 1982. Este proyecto de Punta Larga dio origen a gran cantidad de investigaciones, desde el campo científico, histórico, artístico, religioso y cultural a partir de la vid y la producción vitivinícola en Boyacá, que se encuentran registradas en los diferentes números de la revista Cultura Cientifica.

\section{Conclusiones}

El papel de la Iglesia a nivel educativo siempre ha sido importante primero por su acción evangelizadora durante la colonia, posteriormente como encargado del sistema educativo en gran parte del siglo XIX y en los gobiernos conservadores durante la primera mitad del siglo XX y ahora como protagonista de la educación privada debido al aumento de la demanda social de educación superior, lo que ha llevado a un aumento en la cobertura y al mejoramiento en la calidad del servicio, tal es el caso de la universidad objeto de este estudio.

El Instituto Catequístico Juan de Castellanos ocupó un lugar preponderante dentro del marco de la educación catequística y religiosa como quiera que fuese el primer Instituto Catequístico del país; como Institución de Educación Superior, el Instituto Universitario se especializó en la enseñanza con la metodología a distancia, se preocupó por la capacitación constante de sus directivos, docentes - tutores y de estudiantes, consolidó una fuerte infraestructura a través de convenios interinstitucionales con las diferentes Diócesis del país y con las Secretarias de Educación Municipales.

Su contribución a la Educación Superior a Distancia ha sido de gran importancia, en la actualidad (2011) La Fundación Universitaria Juan de Castellanos hace presencia en más de 60 municipios de 14 departamentos del país bajo ésta modalidad de enseñanza, es una de las de mayor cobertura nacional siendo coherente con su misión institucional ya que la civilización del amor, su slogan, se proyecta al fortalecimiento del campo y la educación del país pensando en la formación como oportunidad de autosostenimiento y emprenderismo según lo manifestaba su fundador el padre Álvaro Castillo Dueñas.

Pese a que sólo lleva medio siglo de existencia la Fundación se ha consolidado, a través de sus programas de pregrado, posgrado, diplomados, capacitaciones y otros cursos de educación continuada como una de las mejores universidades de Boyacá y Colombia. 


\section{Referencias bibliográficas}

Álvarez de Huertas, R. 1998. Entre el gorro frigio y la mitra. La mentalidad políticoreligiosa del hombre neogranadino 1850 - 1887. Tunja: Academia Boyacense de Historia.

Bidegaín de Uran, A. M. 1983. Iglesia, Pueblo y política. Un estudio de conflictos de intereses. Colombia 1930 - 1955. Bogotá: Pontificia Universidad Javeriana.

Cifuentes, M. T. y Figueroa, H. 2004. Corrientes del catolicismo frente a la guerra y la paz en el siglo XX. En: Ana María Bidegaín (dir.). Historia del cristianismo en Colombia. Corrientes y diversidad, P:29. Bogotá: Taurus.

González, Fernán. 2001. La Iglesia Católica y el Estado colombiano (1886-1930). En: Nueva Historia de Colombia. Historia Política 1946 - 1986. Vol . II, P.17. Bogotá: Planeta.

González, Fernán. 1997. Poderes enfrentados. Iglesia y Estado en Colombia. Santafé de Bogotá: Cinep.

Ocampo López, Javier. 1999. Los catecismos politicos en los orígenes de Colombia. En: Iglesia, movimientos y partidos: Política y violencia en la historia de Colombia. Colección Memorias de Historia. P. 36. Tunja: Universidad Pedagógica y Tecnológica de Colombia.

Pablo VI. 1965. Declaración Gravissimum Educationis. Sobre la Educación Cristiana. Roma. 


\section{Fuentes Documentales}

Archivo Histórico. Instituto Universitario Juan de Castellanos.

Castillo Dueñas, Álvaro. Discurso a la primera promoción de graduandos. Somondoco. 10 de diciembre de 1993.

Fundación Universitaria Juan de Castellanos. Consejo Superior. Creación de INICIEN. Acuerdo 095 del 25 de febrero de 1999

Fundación Universitaria Juan de Castellanos. Consejo Superior. Estructura Orgánica. Acuerdo 169 del 1 de noviembre de 2001

Fundación Universitaria Juan de Castellanos. Consejo Superior. Proyecto Educativo Universitario. Acuerdo 220 del 8 de agosto de 2005.

Fundación Universitaria Juan de Castellanos. Consejo Superior. Plan de Desarrollo periodo 2012-2019. Acuerdo 312 del 6 de diciembre de 2011.

Huertas Vargas, José Vicente. Informe del Rector al Consejo Superior. Tunja. 1998

Instituto Universitario Juan de Castellanos. Estudio de factibilidad. Tomo 1. Tunja. 1986

Instituto Universitario Juan de Castellanos. Libro Actas Consiliatura. Informe rectoral del Padre Castillo del 5 de febrero de 1990.

Instituto Universitario Juan de Castellanos. Libro Actas de Reuniones de Directivas. Acta No 3 del 6 de marzo de 1989. 